\title{
Tifones y gigantes en el mundo macedonio: el rey como símbolo del orden cósmico
}

\author{
Antonio Ignacio Molina Marín \\ CEPOAT: Universidad de Murcia \\ miprofeignacio@hotmail.com
}

Recibido: 2 de noviembre de 2014

Aceptado: 8 de mayo de 2015

\begin{abstract}
RESUMEN
Este artículo analiza la existencia de un posible ritual en la antigua Macedonia que fuese similar al Akîtu babilonio. Pensamos que la victoria de Zeus sobre sus enemigos en la gigantomaquia y tifonomaquia fue celebrada por los reyes macedonios para fortalecer su posición en el poder. Aunque no hay evidencias directas de ello, tenemos una serie de diferentes fuentes en las cuales los macedonios están relacionados directa o indirectamente con Tifón y los gigantes. Además, la estratégica importancia del monte Olimpo para los macedonios nos hace pensar que la victoria de Zeus sobre las fuerzas del mal fue conmemorada de alguna forma por ellos.
\end{abstract}

Palabras clave: Akîtu. Altar de Pérgamo. Gigantomaquia. Tifonomaquia. Monte Olimpo. Monte Casio. Seleuco. Alejandro Magno. Antigua Macedonia.

\section{Typhons and Giants in the Macedonian World: The King Like Symbol of the Cosmic Order}

\begin{abstract}
This paper looks at the existence of a possible ritual in the ancient Macedonia that was similar to the Babylonian Akîtu. We think that the victory of Zeus over their enemies in the Gigantomachy and Typhonomachy was celebrated by the Macedonian kings to strengthen their position in the power. Although there is no direct evidence about it, we have a load of different sources in which the Macedonians are related directly or indirectly to Typhon and the Giants. Besides, the strategic importance of Mount Olympus for the Macedonians make us think the victory of Zeus over the forces of Evil was commemorated someway by them.
\end{abstract}

Key words: Akîtu. Gigantomachy. Typhonomachy. Mount Olympus. Mount Cassius. Seleucus. Alexander the Great. Ancient Macedonia.

Sumario: 1. Introducción. 2. Alejandro como combatiente de las fuerzas del caos. 3. Seleuco y el monte Casio. 4. La gigantomaquia y la tifonomaquia en el mundo helenístico. 5. El Olimpo y los reyes de Macedonia. 6. La Xandica y el festival de la Basileia. 7. Conclusión. 


\section{Introducción}

Recientes estudios han puesto en relación el mundo griego con la ceremonia babilonia del año nuevo, Akîtu. ${ }^{1}$ Se ha interpretado la recuperación de este festival por Seleuco y sobre todo por Antíoco I, como un acto de continuidad con la tradición babilónica. Los festejos se celebraban en el mes de Nisán, y durante los mismos se rendía homenaje al triunfo de Marduk frente a Tiamat, una fuerza primigenia del abismo. Durante el noveno día del Akîtu tenía lugar una lucha simulada entre Marduk y Tiamat. Este evento simbolizaría la destrucción y la recreación del cosmos, así como el triunfo del orden frente al caos. No se sabe si el festival retomado por los macedonios en Babilonia fue exactamente igual que el primero, pero no hay duda de que su finalidad fue fortalecer la posición del monarca ante la población de Babilonia al presentarlo como un garante del orden cósmico, ya que durante el festival las personas del monarca y la deidad (Marduk) se confundían. ${ }^{2}$ La utilidad de este ritual para los monarcas provocó que fuese continuado por los persas (Crónica de Nabónido III, 24-28) o que incluso pudieran emularlo en el Now Ruz, su festival de año nuevo. ${ }^{3}$ Ante estos datos no podemos evitar preguntarnos si en Macedonia, ya fuese antes, durante o después de Alejandro, ya fuese autóctono o inspirado por la tradición oriental, existió algún tipo de ritual que asociara al monarca con el orden divino. Encontramos la pregunta necesaria, puesto que además de ser la sede de una dinastía secular, Macedonia fue un lugar destacado en los únicos eventos griegos que pueden compararse a la lucha entre Marduk y Tiamat: la gigantomaquia y la tifonomaquia.

Creemos que con nuestro estudio se podrá valorar mejor si existieron o no indicios de un ritual que sirviera para fortalecer la posición del rey en Macedonia y presentarlo como mediador ante los dioses de su pueblo.

\section{Alejandro como combatiente de las fuerzas del caos}

El mal personificado provoca por igual pavor y fascinación en quien lo contempla. La proyección de los temores y fantasías de un colectivo en un determinado ser parece haber sido un tentación irresistible para todas las culturas. En este hecho se entremezcla la creencia en la existencia de las fuerzas del mal y las del bien. El mal y el bien absolutos parecen haber formado parte de un mismo axioma: cuando el terror se alza también lo hace el campeón que debe derrotar al caos y volver a instaurar el orden que es sinónimo de justicia.

Dado que este mensaje tuvo y tiene una fuerza legitimadora enorme ha sido apropiado tradicionalmente por las monarquías para denigrar a sus adversarios y presentarse directa o indirectamente como los representantes del orden primigenio.

\footnotetext{
1 ERICKSON 2009, 112 y ss.; ID. 2011, 51-63.

2 Erickson defiende muy convincentemente una profunda asociación entre Nabu (Antíoco) y Marduk (Seleuco).

3 Eddy 1961, 51-55; Fennelly 1980, 135-162; García Sánchez 2008, 14 y ss.
} 
¿Se valió Alejandro Magno de este recurso propagandístico? Si alguien ha impulsado la deificación del rey y su asociación con Zeus ha sido Alejandro de Macedonia. Ahora bien, entre las miles de páginas escritas sobre Alejandro no hay ninguna en la que se hable de esta cuestión. No obstante, sí existen algunos indicios que nos permiten introducir al Macedonio en el tema que nos ocupa.

Durante su estancia en Priene, Alejandro inauguró el templo de Atenea Polias. ${ }^{4}$ En dicho templo había una representación de la gigantomaquia. Sin embargo, aunque Alejandro hizo la dedicatoria del templo, costeó sus gastos y puede que hasta conocido su diseño, el proyecto original del mismo proviene de la dinastía Hecatómnida de Caria. ${ }^{5}$

Tanto Alejandro como sus historiadores mostraron un gran interés por la gruta Coricia, la cueva de Tifón: "se podía ver el emplazamiento de las ciudades Lirneso y Tebas, así como la gruta de Tifón y el bosque de Coricio, en donde brota el azafrán". ${ }^{6}$ El texto aunque seguramente procede de Calístenes, ${ }^{7}$ no ha llamado la atención de los estudiosos. No obstante, los historiadores de Alejandro, y en especial Calístenes, solamente destacaban los mitos griegos que tenían especial significancia para su señor. El hecho de que Diogneto de Eretria, un ilustre miembro de los bematistas, ${ }^{8}$ también mencionase a Tifón en su obra (Hyg. Astr. 2.30) parece confirmarlo.

Fredricksmeyer defendió hace unos años que, durante su estancia en Babilonia, Alejandro habría recibido el homenaje de Babilonia mediante el Akîtu. Pese a que las fuentes no lo mencionan y pese a que es cronológicamente imposible, ya que no estuvo en la ciudad durante el mes de Nisán (marzo), sostuvo que recibió una ceremonia supletoria. ${ }^{9}$

El interés de Alejandro por reconstruir el templo de Bel-Marduk era un apoyo importante a la tesis de Fredricksmeyer. ${ }^{10}$ No obstante, al igual que con Apis, la razón pudo haber residido en su conexión genealógica con Belo (Marduk) a través de los mitos de la casa real de Argos. Épafo (Apis) era hijo de Io, y éste al unirse con Menfis tuvo dos hijos: Agenor y Belo (Apollod. II 4.1). De ellos nacerían las Danáides tan estrechamente unidas a Argos, al igual que lo estaba Alejandro a través de su sangre Teménida. Se discute si el Belo egipcio y el babilonio eran el mismo, pero no hay duda de que sus personas se confundieron en la Antigüedad: "Manticlo fundó tam-

4 Para la estancia de Alejandro en Priene Cf. Heisserer 1980, 142-168; sobre la gigantomaquia véase Arena 2013, 59; Manunta 2008, 85.

5 HeISSERER 1980, 157.

6 Curt. III 4.10 (traducción de F. Pejenaute Rubio, Biblioteca Clásica Gredos, 1986). Cf. Str. XIII 4.6.

7 Pearson 1960, 43.

8 Fueron los encargados de medir las distancias recorridas por el ejército. Cf. Molina Marín 2012, 129-130.

9 Fredricksmeyer 2000, 146: "It is not unlikely that, as in Egypt, so also in Babylon Alexander had himself formally consecrated as king. There may have been two such ceremonies for the Babylonian kings. One, at the New Year's Festival (Akîtu) when Bel-Marduk confirmed, or reconfirmed, the candidate in the kingship. The festival took place in the spring (March), while Alexander left Babylon no later than early December (331). Perhaps Marduk's priests arranged a substitute ceremony for Alexander. As the New Year's Festival legitimated the kingship on an annual basis, there may have been another ceremony for the initial inauguration of the king. Alexander's sacrifice to Bel-Marduk, reported by Arrian (3.16. 5), may have been part of this inauguration ceremony".

10 Arr. An. III 16.4 y VII 17.1-2; Str. XVI 1.5. 
bién el santuario de Heracles entre los mesenios, y fuera de la muralla está instalado el dios llamado Heracles Manticlo, como Amón en Libia y Belo en Babilonia, que recibió el nombre por un egipcio, Belo, hijo de Libia, y Amón por el pastor que lo construyó". ${ }^{11}$ Alejandro tenía suficientes motivos propios para sentir curiosidad por Belo. Otra cuestión es si pudo conocer la lucha entre Marduk y Tiamat y haber considerado a Belo como un "dragonslayer" semejante a Heracles y Zeus. En cualquier caso, la posibilidad de una interpretatio graeca es factible y podría haber sido un elemento importante para avivar el interés de Alejandro por templo de Marduk. ${ }^{12}$

No existen otros vínculos directos entre Alejandro y el combate contra las fuerzas del caos en las fuentes. No obstante, creemos que el tesoro de Poro puede ser esgrimido como una probable representación de una gigantomaquia. Se han dado muchas interpretaciones sobre estas monedas. Bosworth defendió que el objetivo real de las mismas habría sido asustar a las póleis griegas. ${ }^{13}$ Goukowsky vio en este hecho el paso definitivo en la deificación de Alejandro Magno. ${ }^{14}$ Holt sostiene que son de época de Alejandro, pero las considera monedas conmemorativas, no destinadas a realizar transacciones comerciales y, por lo tanto, con una difusión menor de la que podría presuponerse. ${ }^{15}$

No obstante, hay un hecho en el decadracma que creemos interesante resaltar, y es el mayor tamaño de los indios respecto al jinete identificado con Alejandro. Tal es su envergadura que se asemejan a gigantes. ${ }^{16}$ Las fuentes destacaron la altura de Poro, y lo retrataron como un auténtico coloso. ${ }^{17}$ Del rey Sopites también se resalta su altura de cuatro codos. ${ }^{18} \mathrm{Si}$ Ctesias de Cnido tenía razón sobre el gigantismo de los seres vivos de la India, su teoría era igualmente extrapolable a los habitantes de la región. Los hombres que montaban elefantes como si fuesen caballos debían ser vistos como gigantes. En suma, es probable que la batalla del Hidaspes fuese presentada ante el mundo griego como una gigantomaquia, ${ }^{19} \mathrm{y}$ un triunfo heroico de Alejandro sobre las fuerzas de la naturaleza.

De este modo, se entendería cómo pudo ser una creencia generalizada la idea de que el elefante de la India era mayor que el africano o por qué los gigantes fueron asociados con esta región. ${ }^{20}$ En este sentido resulta llamativa la historia contada por

11 Paus. IV 23.10 (traducción de M. C. Herrero Ingelmo, Biblioteca Clásica Gredos, 1994).

12 Bernard 1990, 525-528, los documentos epigráficos conservados desprenden un interés personal de Alejandro por Belo.

13 Bosworth 1996, 8.

14 Goukowsky II 1981, 3.

15 Holt 2003, 54.

16 Holt 2003, 129.

17 Plut. Alex. 60.12; Arr. An. V 19.1; Diod. XVII 88.4; Curt. VIII 14.13.

18 Diod. XVII 91.7.

19 Cf. Koepp 1888, capítulo III; Hardie 1985, 86; Molina Marín 2014, 87.

20 Paus. VIII 32.5: "El Orontes, que es un río de los sirios que corre no todo en llano hasta el mar, sino sobre un precipicio escarpado, y desde él se precipita en cuesta, el emperador romano quiso que fuera navegable desde el mar hasta la ciudad de Antioquía. Entonces cavó con trabajo y gasto de dinero un canal apropiado para la navegación corriente arriba, y desvió el río hacia éste. Pero cuando se secó el antiguo cauce, se encontró en él un ataúd de barro de más de once codos, y el muerto tenía el mismo tamaño que el ataúd y era humano en todas las partes de su cuerpo. Cuando los sirios fueron al oráculo, el dios de Claro les dijo que era Orontes, de la raza de los indios" (traducción de M. C. Herrero Ingelmo, Biblioteca Clásica Gredos, 2008). 
la Vulgata en la que Alejandro ordenó construir un campamento de dimensiones colosales con la intención de hacer creer a las generaciones venideras que la India había sido conquistada por una raza de gigantes. ${ }^{21}$
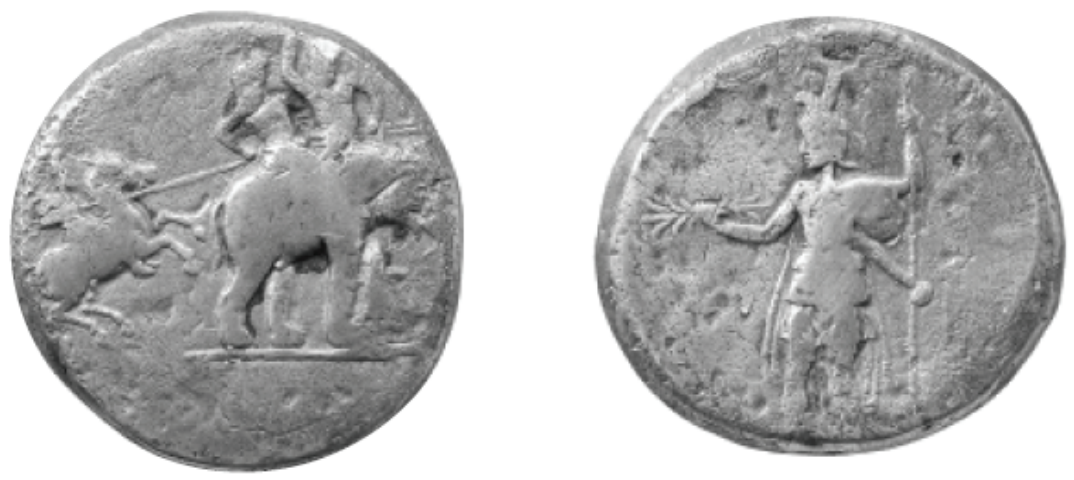

Fig. 1. Decadracma de Poro (Holt 2003, plate 4).

Plutarco en los Moralia relaciona de forma indirecta a Alejandro con Tifón y los

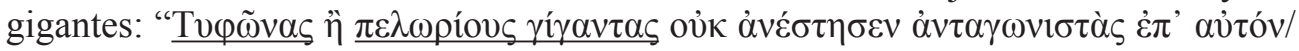
¿Qué Tifones o gigantes monstruosos no levantó (la Fortuna) para oponerse a él?". ${ }^{22}$ La cita tal vez fuese algo más que un simple retoricismo del autor de Queronea ${ }^{23}$ ¿Quiso el Macedonio, descendiente de Heracles e hijo de Zeus Amón emular a su divino linaje en otro aspecto más de sus vidas? ${ }^{24}$ Disputar una lucha contra las fuerzas del caos. ${ }^{25}$

\section{Seleuco y el monte Casio}

Poco después de la batalla de Ipsos (301 a.C.), Seleuco afianzó su poder en Siria con la fundación de su famosa tetrápolis. La monarquía Seléucida inició un proceso de macedonización del territorio sirio que se plasmó con el traslado de topónimos muy

21 Diod. XVII 95.2; Curt. IX 3.19; Justin. XII 8.16.

22 Plut. De Alex. Fort. 341E (traducción propia).

23 El pasaje de Plutarco se inspira en este otro de Eurípides (Heracl. 1270-5): “ ¿a qué enumerar los trabajos que soporté; el número de leones, tifones de tres cuerpos, gigantes o ejércitos de cuadrúpedos centauros a quienes no declaré la guerra?" (traducción de J. L. Calvo Martínez, Biblioteca Clásica Gredos, 1985). Podría ser un añadido de Plutarco para asemejar las hazañas de Alejandro con los trabajos de Heracles, pero dada la devoción de Alejandro por su antepasado también pudo proceder de una fuente usada por Plutarco.

24 Sobre la emulación de Alejandro hacia sus ancestros divinos cf. Edmunds 1971; ANTEla Bernárdez 2007; Molina Marín 2012a.

25 En la leyenda de Alejandro se encuentra con algunos gigantes según la recensión alpha del PseudoCalístenes (II 33): "Y desde aquí partimos y llegamos a una región herbosa en la que existían unos hombres salvajes con figura de gigantes, esféricos, de rostro rojo y aspecto leonino" (traducción de C. García Gual, Biblioteca Clásica Gredos, 1988). 
conocidos de Macedonia: ${ }^{26}$ Edessa, Pela, Anfípolis, Beria, Botia, Europos, etc. El río Orontes fue bautizado con el nombre del Axios, uno de los ríos más importantes del antiguo reino. ${ }^{27}$ Rastros de los antiguos mitos de Argos, tan ligados a los Teménidas, fueron rastreados y localizados en suelo sirio. No hay duda que de que Seleuco estaba creando una Macedonia en miniatura, ${ }^{28}$ y que el universo mental macedonio fue recreado para dar respuesta a las necesidades de los colonos, que iban más allá de la simple nostalgia. ${ }^{29}$ Malalas nos da un ejemplo de este proceso:

Inmediatamente después de su victoria sobre Antígono Poliorcetes, ${ }^{30}$ Seleuco Nicator quiso edificar una serie de ciudades y comenzó primero a fundar en la costa siria. Bajando al litoral vio en una montaña una pequeña ciudad que era llamada Paleópolis, que Siros, el hijo de Agenor, había construido. El 23 del mes de Xandicos fue al monte Casio para sacrificar a Zeus Casio. Después de completar los sacrificios y cortar la carne, preguntó en una oración dónde debía fundar una ciudad. De repente, un águila agarró algo del sacrificio y se lo llevó fuera de la vieja ciudad. Seleuco y los augures la siguieron de cerca y encontraron la carne arrojada junto al mar, cerca del enclave comercial conocido como Pieria. Después de trazar los muros, estableció inmediatamente su fundación, llamándola Seleucia como él mismo. Para dar gracias fue a Iópolis, donde tres días después celebró un festival en honor de Zeus Ceraunio, en el templo construido por Perseo, hijo de Pico y Dánae, que está en el monte Silpio, donde se sitúa Iópolis. Hizo el sacrificio el primero del mes de Artemision. ${ }^{31}$

La ciudad de Iópolis parece haber estado relacionada con el mito de Io, ${ }^{32}$ que al igual que Perseo formaban parte de la genealogía de Argos. Su inclusión en esta historia debe ser entendida como un hecho más en el proceso de macedonización de Siria, y también como una forma de vincular a los Seléucidas con la casa real de Macedonia, que como sabemos pretendía proceder de Argos. ${ }^{33}$

Sin embargo, el objetivo de la historia parece haber sido más ambicioso. Los acontecimientos se repiten cuando Seleuco consulta al oráculo sobre la idoneidad de cambiar el nombre de la ciudad de Antigonea o si debía establecerse en otro sitio: un águila desciende del cielo y vuelve a coger un pedazo de la carne sagrada del sacrificio. ${ }^{34}$ Se entiende que dicho animal es un símbolo de Zeus, divinidad a la que Seleuco de-

26 Grainger 1990, 42; Antela Bernárdez 2009, 124.

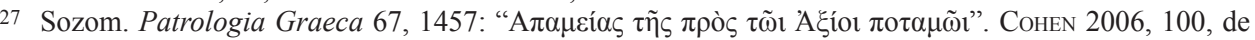
hecho el topónimo Axios puede ser encontrado en algunos geógrafos árabes bajo la forma de al-Asi.

28 Billows 1995, 146: "Seleukos turned northern Syria into a new Macedonia"; AnTEla BernáRdez $2009,124$.

29 LANE Fox 2008, 112.

30 Hay una confusión evidente entre Antígono Monoftalmos y Demetrio Poliorcetes.

31 Malalas VIII 199 (traducción propia).

32 Libanio (Or. XI 61) llama a la ciudad Ione. Cf. Downey 1961, 51: "The legends might have arisen in part in connection with temples that could have come to be identified with those supposed to have been built at Iopolis, and the annual commemoration of the search for Io, which Malalas says the people of Antioch later observed, might have grown out of an oriental rite that came to be associated with the commemoration of Io's wandering".

33 Hdt. VIII 138.

34 Libanio (Or. XI 85-8) cuenta una historia similar, pero en su relato no es Seleuco quien persigue el ave, sino su hijo Antíoco. 
dicaba sus ofrendas bajo los apelativos de Zeus Casio y Ceraunio ("rayo"). El águila que capturaba a su presa entre sus garras parece haber sido un símbolo iconográfico rastreable también en el Egipto de los Ptolomeos ${ }^{35}$ y en la propia Macedonia. ${ }^{36} \mathrm{De}$ hecho, Posidipo (31), dice que para los macedonios un águila era un signo favorable en la guerra. ${ }^{37}$

Tras una nueva persecución vuelven a encontrar el trozo de carne en el lugar escogido por los dioses para albergar la nueva ciudad de Seleuco. Lo llamativo es que el emplazamiento está "cerca del gran río Drakon, rebautizado como Orontes, en el lugar de la aldea conocida como Botia". ${ }^{38}$

Como hemos dicho anteriormente se conoce un tercer nombre para el Drakon/ Orontes: el Axios. ${ }^{39}$ De este modo algunos de los principales topónimos y lugares que Tucídides (II 99) mencionaba en su pasaje sobre la evolución del reino de Macedonia podían encontrarse en la tetrápolis de Seleuco: Pieria, Pela, Botia y el Axios. Este último era especialmente importante, ya que era el río que regaba el corazón del reino y su capital Pela. Llama la atención el nombre originario del Orontes: Drakon. Esta palabra puede significar en griego tanto serpiente como dragón, y posiblemente se deba a los meandros que el río traza en su curso..$^{40}$ Estrabón da una explicación sobre el origen del término drakon y añade una nueva denominación a la lista:

Aunque anteriormente llamado Tifón, su nombre fue cambiado a Orontes, el hombre que construyó un puente a través de él. Aquí, en alguna parte, se sitúa la historia mítica de los Árimos, de quienes ya he hablado. Dicen que Tifón, que añaden que era un dragón, cuando fue golpeado por los relámpagos, huyó en busca de un refugio subterráneo; de modo que no sólo cortó la tierra en surcos y formó el lecho del río, sino también marchó bajo tierra y originó que la fuente brotase a la superficie. ${ }^{41}$

Ahora entendemos que el nombre del río está conectado con una tifonomaquia, y que tanto el monte Casio como Zeus Ceraunio deben analizarse en ese contexto, puesto que el Drakon/Tifón/Orontes/Axios estaba detrás de la cumbre. La tifonomaquia no era ubicada allí únicamente por los griegos, sino también por indígenas. Se piensa que pudo haber sido el escenario de la lucha entre Baal y Lotán, ${ }^{42}$ el equivalente del hebreo Leviatán y de Tiamat en la religión babilonia.

Píndaro ( $P$. I 15-28; VIII 15-16) situó la lucha entre Tifón y Zeus cerca de Cilicia, pero la primera fuente que menciona el monte Casio es Pseudo-Apolodoro (I 6.3) es decir, siglos después del reinado de Seleuco. En suma, podría pensarse que todo el

35 Eliano y la Suda (s.v. Lágos=Aelian F 283) dicen que Ptolomeo fue protegido por un águila poco después de nacer, puesto que fue expuesto en un escudo de bronce por su padre Lago. El ave también se encuentra en las monedas de varios Ptolomeos.

36 GAEBLER 1935, plates xxix.16, xxx.1.7 y 17.

37 Recuérdese que antes de la batalla de Gaugamela, Alejandro también se habría sentido respaldado por los dioses al contemplar un águila (Plut. Alex. 33.2-3).

38 Malalas VIII 200 (traducción propia).

39 Cf. Grainger 1990, 42.

40 OGden 2013a, 35.

41 Str. XVI 2. 7 (traducción propia). Cf. Str. XVI 2.17.

42 Ogden 2011, 94. 
relato no sería más que otra interpretatio graeca de un mito local. Ahora bien, eso sería simplificar las cosas. En nuestra opinión la tifonomaquia es otro elemento en el proceso de macedonización de Siria. Si los topónimos y el río jugaban una parte importante en el desarrollo de los acontecimientos, el monte Casio (Jebel Aqra) es el auténtico motor del mismo y la verdadera razón de que se escogiera el emplazamiento para fundar la tetrápolis de Seleuco, ya que está asociado con el corazón de Macedonia, el monte Olimpo. ${ }^{43}$

El que el monte Casio desempeñó para Seleuco y sus colonos el rol que el Olimpo tuvo para los macedonios, parece confirmarlo la presencia de otros mitos en la zona ligados con los gigantes, los otros famosos asaltantes del Olimpo:

Seleuco levantó una estatua de un águila justo fuera de la ciudad. Ordenó que los meses en Siria debían ser llamados según la costumbre macedonia, dado que descubrió que los gigantes habían vivido en la región. A dos millas desde la ciudad de Antioquía hay un lugar con cuerpos humanos convertidos en piedra a causa de la cólera de Dios, que son llamados gigantes hasta el día de hoy. De igual modo, un gigante conocido como Pagras, que vivió en la tierra, fue calcinado por un rayo. Así que todo indica que el pueblo de Antioquía en Siria vivió en la tierra de los gigantes. ${ }^{44}$

Es sumamente, difícil afirmar si Seleuco ordenó adoptar por completo el calendario macedonio en Siria o si fue una mezcla de ambos calendarios. ${ }^{45}$ Por otro lado, autores como Adrienne Mayor ${ }^{46}$ han dado validez al pasaje de Malalas, y han considerado que lo que aquí se estaba describiendo no era más que el hallazgo de fósiles antiguos. Ahora bien, la presencia de los gigantes junto a la tifonomaquia anteriormente descrita, reconstruían una realidad que se encontraba en Macedonia en la zona del monte Olimpo y que por algún motivo era perentorio y necesario para Seleuco trasladar a Siria.

\section{La gigantomaquia y la tifonomaquia en el mundo helenístico}

Uno de los ejemplos más bellos de esta concepción maniquea que estamos estudiando es el Altar de Zeus en Pérgamo. ${ }^{47}$ Es sobradamente conocido que los reyes de este reino comisionaron un altar para celebrar sus victorias sobre sus enemigos. No es nuestro objetivo el estudio pormenorizado de esta monumental obra, tan sólo incidir en lo ya comentado: los adversarios de los reyes son asociados con seres monstruosos, gigantes. Por el contrario, los dioses olímpicos son el alter ego de los reyes de

43 Lane Fox 2008, 112: "Jebel Aqra, the seat, like a local Olympus, of the ruling god whom the Greeks identified as Zeus".

44 Malalas VIII 202. Queremos mostrar nuestro agradecimiento con Daniel Ogden por habernos indicado la relevancia de este pasaje.

45 TARN 1938, 64: "It used to be taken for granted that, where the names of the Macedonian months occur, the Macedonian form of the calendar was in use, but recently it has been strongly argued that the calendar in use at Seleuceia was a mixed form, with the Macedonian months but the Babylonian year".

46 MAYOR 2000, XX.

47 Pollitt 1989, 168-185. 
Pérgamo. La gigantomaquia servía a la vez para denostar a los rivales de la casa Atálida y para glorificar al vencedor. ${ }^{48}$

No obstante, hay un hecho sobre el que queremos llamar la atención y es la identidad de los gigantes derrotados. Se cree que el monumento conmemora la victoria sobre los gálatas del 166 a.C., quienes por su tamaño y altura invitaban a ser relacionados con auténticos gigantes. ${ }^{49}$ El Altar de Zeus donde se representaba la gigantomaquia debía ser visto como el símbolo de la victoria Atálida frente a sus rivales. Ahora bien, si los gigantes eran una personificación de los gálatas, ¿por qué aparecen representados portando escudos macedonios con la estrella de los Argéadas? Este símbolo estaba incuestionablemente conectado con los reyes de Macedonia, y posiblemente hasta los tiempos de Alejandro sólo podían lucirlo los miembros de la casa real Teménida o Argéada. ${ }^{50}$ Introducirlo en una gigantomaquia no podía ser un hecho fortuito. Otros elementos característicos de la iconografía macedonia como la anastole, el famoso peinado de Alejandro, ${ }^{51}$ nos llevan a confirmar la existencia de una relación entre los gigantes y el reino de la antigua Macedonia. ${ }^{52}$

Hay que tener presente que pese a que las primeras manifestaciones artísticas de la victoria de los Atálidas se centraron en los gálatas, el Gran Altar de Zeus fue construido entre el 181 y el 159 a.C., es decir, después de la guerra entre Macedonia y Roma. Los Atálidas lucharon en el bando romano y pudieron utilizar el recurso de la gigantomaquia para conmemorar su triunfo. Ahora bien, ¿por qué se representa a los vencidos como gigantes?

Además de ser criaturas malignas, eran una encarnación de la soberbia y de la inmoderación, de la más pura hybris, ${ }^{53}$ ya que intentaron invertir el orden cósmico asaltando el monte Olimpo. Una gigantomaquia encajaba con la voracidad de un pueblo como el macedonio que había conquistado casi todo el mundo conocido, pero que había sido aplastado por el peso de su propia ambición. Por otro lado, una de las versiones sobre el origen de los gigantes establecía que habían nacido en una región de la Calcídica, Palene, ${ }^{54}$ es decir, en suelo macedonio.

El éxito de este recurso queda atestiguado por su difusión en las artes, ${ }^{55}$ pero la representación de los enemigos de una dinastía como las fuerzas de la oscuridad no fue una innovación de los reyes de Pérgamo, ni el gran Altar la única conexión de los macedonios con los gigantes.

48 Hardie 1986, 129; Whitaker 2005, 167: "I think there can be no doubt that the viewer was meant to connect the gods' defeat of the giants with the Attalid defeat of the Galatians".

49 Callaghan 1981, 115-121; Stewart 2000, 34; Whitaker 2005, 164-165.

50 Tripodi 1986, 658; LiAMPI 1990.

51 STEWART 1993, 45.

52 Ridgway 2001, 121. Cf. Trofimova 2012, 125-132, quien dice que tanto vencedores como vencidos presentan rasgos de la iconografía de Alejandro Magno, por lo que no podría hablarse de una propaganda antimacedonia. Sin embargo, la autora no explica por qué los gigantes están armados con sarisas y escudos macedonios.

53 Pi. $P$. VIII 1-8.

54 Paus. I 25.2-3. Str. VII 25. Cf. Paus. VIII 29.1. Por el contrario, Píndaro (N. I 67) localiza su nacimiento en los campos Flegreos (Sicilia). Cf. Hdt. VII 127.

55 Hardie 1986, 85; Dowden 2006, 38-39. 
El poeta Alceo de Mesene escribió unos versos en los que Filipo V de Macedonia era comparado con un gigante: "Zeus Olimpio, tus muros levanta, que todo a Filipo le es accesible $(\hat{\alpha} \mu \beta \alpha \tau \alpha \dot{)})$; cierra tus broncíneos portales. Ya el poder de Filipo domina los mares y tierras; ya sólo la conquista te queda del Olimpo". ${ }^{56}$ Se ha discutido mucho si las palabras de Alceo fueron un elogio o un ataque a Filipo, ${ }^{57}$ pero el término $\alpha \mu \beta \alpha \tau \alpha ́$ está muy ligado a otros intentos de escalar el Olimpo por monstruos dominados por su ambición. ${ }^{58}$ Así que Filipo es representado como un usurpador y un rebelde contra el orden establecido.

Ovidio y Antonino Liberal recrean la llamada Musomachia, el duelo entre las musas Piérides y las musas del Helicón por la supremacía de las artes. Piérides es un término usado como sinónimo de las musas en Macedonia y su conexión con la región era incuestionable. ${ }^{59}$ En la versión de Ovidio las Piérides ensalzan a los gigantes y a Tifón como los verdaderos vencedores en la lucha contra las deidades del Olimpo. ${ }^{60}$ Las musas macedonias tienen mucho en común con los asaltantes del monte, ya que intentan echar a las musas del Helicón y ganar la supremacía, pero su soberbia les lleva a la perdición como a ellos. La fuente común de Ovidio y Antonino Liberal es Nicandro, ${ }^{61}$ un autor del siglo II a.C., que se cree que pudo haber reflejado la propaganda antimacedonia de la corte de Pérgamo en sus escritos. ${ }^{62}$

En el llamado Oráculo del alfarero, siglo III a.C., podemos leer como los portadores del cinturón, la élite greco-macedonia que gobierna Egipto, es vinculada a Tifón: “....los portadores del cinturón se aniquilarán a sí mismos porque son seguidores de Tifón. Entonces Agathos Daimon abandonará la ciudad que se está edificando (Alejandría) y emigrará y quedará desierta la ciudad de extranjeros que se está construyendo entre nosotros" (traducción propia). En este caso los paralelismos son casuales, ya que desde los tiempos de los hicsos los extranjeros estaban estrechamente unidos en la mentalidad egipcia a Seth, ${ }^{63}$ y esta deidad era la equivalente en griego a Tifón. ${ }^{64}$

56 AP IX 518 (traducción de M. Fernández Galiano, 1978).

57 EdSON 1934, 214: elogio; WALBANK 1940, 120: "sarcastic verses"; MomigLiano 1942, 53: "the ironical intention does not appear in the text and has been assumed only because Alcaeus wrote some epigrams against Philip V"; Walbank 1944, 88: "praise"; Edson 1948, 119: "ironical".

58 El caso paradigmático es el de los Alóadas: Oto y Efialtes (Od. XI 316). Cf. Edson 1948, 118-119.

59 Procede de Pieria, región de la Baja Macedonia (Il. XIV 226) conocida por ser el hogar de las musas (Hes. Th. 53; E. Med. 832). Cf. GiRTzy 2001, 83-86; Mallios 2011, 82-91.

60 Ov. Met. 315-30: “"Turpe quidem contendere erat, sed cedere visum turpius; electae iurant per flumina nymphae factaque de vivo pressere sedilia saxo. tunc sine sorte prior quae se certare professa est, bella canit superum falsoque in honore gigantas ponit et extenuat magnorum facta deorum; emissumque ima de sede Typhoea terrae caelitibus fecisse metum cunctosque dedisse terga fugae, donec fessos Aegyptia tellus ceperit et septem discretus in ostia Nilus. huc quoque terrigenam venisse Typhoea narrat et se mentitis superos celasse figuris; "duxque gregis" dixit "fit Iuppiter: unde recurvis nunc quoque formatus Libys est cum cornibus Ammon; Delius in corvo, proles Semeleia capro, fele soror Phoebi, nivea Saturnia vacca, pisce Venus latuit, Cyllenius ibidis alis".

61 Johnson - Malamud 1988, 31.

62 Mallios 2011, 85-91.

63 Man. FgrH 609, F. 10 (237).

64 Burstein 1985, 106. 
Ahora bien, ni Pérgamo fue el único reino en personificar a sus enemigos como la encarnación de las fuerzas del mal, ${ }^{65} \mathrm{ni}$ los Sucesores de Alejandro parece que hayan pasado por alto el empleo de esta estrategia. Ilustres miembros de las casas reales helenísticas pudieron haberse presentado como vencedores de gigantomaquias durante el mundo helenístico. Antíoco I tal vez celebró su triunfo frente a los gálatas de esa manera. ${ }^{66}$ Calímaco (IV 170-5) compara a los gálatas con los Titanes que asolaron Grecia y más tarde fueron derrotados por Ptolomeo II Filadelfo. El Himno a Zeus de Calímaco es tanto un elogio al dios como a Filadelfo, ${ }^{67}$ puesto que si Zeus derrotó a los Pelagones, es decir, a los gigantes que querían usurpar su poder, Ptolomeo II hizo lo propio con su hermanastro Ptolomeo Cerauno, quien adoptó el sobrenombre de Cerauno ("el rayo") con intenciones posiblemente muy similares, ya que era con este arma con la que el padre de los dioses derribaba a quienes pretendían destronarle.

Este tipo de comparación fue más fácilmente asumible conforme la asociación entre el monarca helenístico y Zeus se hizo más profunda con el desarrollo del culto al gobernante. De tal modo que los derrotados por el monarca podían ser comparados con gigantes, no sólo por su tamaño sino ante todo por aspirar de forma ilegítima al poder, pues al igual que Zeus gobernaba sobre los dioses, el rey debía hacerlo sobre los hombres (cf. Call. Jov. 79-85).

\section{El Olimpo y los reyes de Macedonia}

El monte Olimpo fue el hogar de los dioses y la sede de las dos luchas míticas que estamos estudiando: la gigantomaquia y la tifonomaquia. La especial relevancia del Olimpo para los macedonios queda atestiguada por ser la sede de su ciudad santuario: Díon. ${ }^{6}{ }^{6}$ El nombre es inequívoco, ya que hace alusión a Zeus, padre de los dioses y señor del Olimpo. ${ }^{69}$ No se sabe mucho de este santuario antes de que Arquelao I estableciera allí un festival dedicado a las musas y que también incluía juegos en honor de Zeus. ${ }^{70}$

Nuestro conocimiento sobre el festival no va más allá de lo expuesto. En cualquier caso, sabemos que, como en todos los actos religiosos, el rey debió tomar parte activa en el ritual, ya que era el máximo sacerdote (Cf. Arr. An. VII 25.2). La impronta de Zeus en el santuario y en la vida diaria de Macedonia no puede ser subestimada, de hecho, Dios, el primer mes del calendario macedonio y equivalente a nuestro mes de

65 La dinastía Dinoménida habría utilizado a Tifón como un símbolo de su triunfo sobre los cartagineses y los etruscos. Cf. Pi. P. I 13-28.

66 HaRdie 1986, 86-87.

67 Clauss 1986, 162.

68 Thuc. IV 78.6. Cf. HARRINGTON 1996, 28: "How and why Dion became a sacred city is unknown, though most scholars assume its proximity to Mount Olympus, the home of Zeus and the Greek pantheon, led to its development as a sanctuary"; PANDERMALIS 1997, 9.

69 Justin. XXIV 2.8.

70 Diod. XVII 16.3-4; Hammond - Griffith II 1979, 149; BorZA 1990, 167; ERrington 1990, 223; Pingiatoglou 2010, 190; Mari 2011, 92; ID. 2011a, 456: “According to our sources, the festival was founded (or rather perhaps reorganized) by King Archelaus (413-399 bc) as a panegyris similar to those celebrated in the main sanctuaries of southern Greece". 
octubre, llevaba su nombre en su honor. ${ }^{71}$ Además, no hay que olvidar que al ser descendientes de Heracles, los Argéadas también lo eran de Zeus. ${ }^{72}$ Su culto está más que atestiguado en la zona con una variedad de epítetos que demuestran su importancia y sus diferencias respecto a las póleis griegas. Un ejemplo fue Zeus Hetairos, mediante su culto (Hetairideia), el rey de Macedonia y sus hetairoi (compañeros) creaban lazos religiosos entre ellos. ${ }^{73}$ De entre todas las manifestaciones de Zeus, sobresalía la de Zeus Basileus (Zeus Rey) tan íntimamente ligado a la función del dios como soberano. La relación entre el rey del Olimpo y el de Macedonia parece haber ido en aumento desde el reinado de Arquelao. A partir de ese momento, el águila, símbolo de Zeus, estará presente en las acuñaciones de Arquelao, Amintas III y Pérdicas III. Filipo II fue synnaos theos del dios en Éreso, ${ }^{74} \mathrm{y}$ aunque como afirma Nock no puede leerse como una evidencia de la deificación de Filipo, ${ }^{75}$ sí lo es de su especial conexión con Zeus, ya que se trató del primer caso conocido de un mortal como synnaos del rey de los dioses. El día de su muerte una estatua de Filipo acompañó a otras de los 12 dioses en una procesión (Diod. XVI 92.5). Alejandro no se contentó con asociarse a través de las monedas, ${ }^{76}$ y proclamó oficialmente su filiación como hijo de Zeus (Plut. Alex. 28.2-3; Arr. An. IV 9.9; VII 8.3). Casandro dedicó una estatua de bronce del dios en Díon que por mucho tiempo se pensó que era una imagen del monarca. ${ }^{77} \mathrm{El}$ águila de Zeus siguió presente en las monedas de la casa Antigónida, ${ }^{78}$ siendo su vinculación con el dios un nexo común de todas las dinastías de la historia de Macedonia.

Zeus parece haber estado muy ligado a la montaña en Grecia y especialmente en el culto macedonio. Zeus akraios, el Zeus de las alturas, fue adorado tanto por los macedonios como por los magnesios. ${ }^{79}$ Alejandro hizo sacrificios desde la cumbre de una de ellas antes de Issos (Curt. III 8.22) y ya hemos visto cómo Seleuco hizo lo propio en el monte Casio. Filipo V ascendió al monte Hemo sin que se conozcan muy bien sus motivos. ${ }^{80} \mathrm{Si}$ hay una montaña unida íntimamente al nombre de Zeus es el Olimpo, dado que fue el símbolo de su poder y el lugar desde el que había repelido los intentos de destronarlo, ya que la montaña era su trono. ${ }^{81}$ Resulta sorprendente que los macedonios no conmemorasen este hecho de alguna forma tal y como hicieron los babilonios, pero no hay evidencias escritas, epigráficas o arqueológicas de un ritual

71 KALLÉRIS II 1976, 557.

72 Le Bohec-Bounet 2002, 42: "The kings of Macedon considered themselves to be descended from Zeus".

73 Le Bohec-Bouhet 2002, 44; Engels 2010, 97.

74 IG XII 2.526. Cf. Heisserer 1980, 27-78.

75 Nock 1930.

76 Le Rider 2007, 11-12; Dahmen 2007, 11.

77 Palagia 2011, 490.

78 Mørkholm 1991, 164.

79 Engels 2010, 97.

80 Liv. XL 21.2: "Le habían entrado ganas de subir hasta la cima del monte Hemo, porque había dado crédito a la opinión común de que desde allí se podía divisar a la vez el Ponto, el mar Adriático, el río Histro y los Alpes" (traducción de J. A. Villar Vidal, Biblioteca Clásica Gredos, 1993).

81 Hom. Il. I 589: “señor del Olimpo"; XXI 505: "Olimpo, a la casa de Zeus de broncíneos muros”; Od. II 68: "el señor del Olimpo"; Pi. N. I 12: "Señor del Olimpo"; O. II 12: "el asiento del Olimpo dominas"; IX 57: "el señor del Olimpo”; fr. 36: “¡Ammón, Señor del Olimpo!”. 
o ceremonial semejante al Akîtu en Macedonia. Sin embargo, sí que podemos ver huellas del paso de los gigantes y Tifón en la geografía macedonia, pues parece ser un rasgo de estas criaturas el dejar recuerdo de sus terribles combates en el paisaje. ${ }^{82}$

Oto y Efialtes modificaron sustancialmente la orografía de los alrededores del Olimpo al utilizar el Osa y el Pelión para escalar el hogar de los dioses (Hom. Od. XI 315-6; Apollod. I 7.4; Hyg. Fab. 28).

Atos fue el nombre de uno de los gigantes que desafiaron a los dioses durante la gigantomaquia. La roca que arrojó contra Poseidón acabó por convertirse en el famoso monte. ${ }^{83}$ Es conocida la historia de cómo el arquitecto Dinócrates de Rodas le ofreció a Alejandro (Plut. Mor. 335 c-d.) moldear el monte con su efigie. En sus cercanías Alexarco, uno de los hermanos de Casandro, fundaría la utópica comunidad de Uranópolis (Str. VII fr. 35).

El gigante Tifoeo, que no debe ser confundido con Tifón, arrasó las montañas de Ematia (Macedonia) durante su lucha con los dioses (Nonn. D. 48.6).

No hay que olvidar que en la gigantomaquia fue igualmente importante otro antepasado argéada: Heracles. A los dioses se les había vaticinado que no podrían aniquilar a ningún gigante a menos que un mortal combatiera a su lado. ${ }^{84}$ De modo que Heracles hería con sus flechas a los gigantes que Zeus y los dioses terminaban matando ¿Puede ser visto en este mito Heracles como un alter ego del rey de Macedonia?

El monte Olimpo fue el punto de inicio del terrible combate entre Zeus y Tifón (Hes. Th. 855-60), una lucha que daría la vuelta al mundo y que cambiaría aún más el espacio. En Macedonia y Tracia dejaron la huella de su paso en el monte Hemo (la cordillera de los Balcanes), que se llama así debido a la sangre de Tifón que cubrió el monte (Apollod. I 6.3). Nono también describe cómo los rayos de Zeus calentaban los acuíferos de la garganta de Migdonia, llenando de vapor toda el área (Nonn. D. I 145-153).

En definitiva Tifón y los gigantes fueron los creadores de una nueva geografía en el paisaje de Macedonia. Era imposible no recordar alguno de estos dos grandes enfrentamientos al contemplar el territorio macedonio, por lo que el área alrededor del Olimpo debió quedar asimilada al recuerdo de estas dos grandes luchas.

Si bien es cierto que la mayoría de nuestras fuentes son posteriores a Alejandro Magno, algunas de ellas pueden estar apoyándose en testimonios más antiguos. ${ }^{85}$ No hay que pasar por alto que el Olimpo fue el escenario de otra usurpación según la religión órfica, antes del triunfo de Zeus y de sus hermanos: la realizada por Cronos contra Eurínome y Ofión, quienes fueron los primeros señores del monte. ${ }^{86}$

82 Ogden 2013a, 23: "A recurring feature of terrible dragon fights is that they leave permanent memorial of themselves behind in the landscape".

83 Nicandro $26(20)=$ Eust. 980.43.

84 Apollod. I 6.1. Cf. Hes. Th. 954. Kertész 1993, 676, destaca que Heracles tiene en Macedonia y en el Altar de Pérgamo un gran protagonismo: "Also the Gigantomachy scene of this altar gave Heracles an important role when showing the victory of Gods over the Giants, ascribing the main merit to Heracles... The intention of the creators is evident; they wanted to exhibit the descent of the Attalids from Heracles. (As we know the kings of the ancient Macedonia, the Temenids had done the same)".

85 OGden 2013, 78: "But these later texts surely do derive much from earlier ones. The scholium to Oppian cites the Titanomachy (genuinely), and Apollodorus may derive his material from a source of some antiquity".

86 Apoll. Rhod. Argon. I 503ss; Origen. Contra Celsum VI 43. 
Nos resulta, por lo tanto, difícil aceptar que en la mentalidad macedonia su espacio fuese un recordatorio de las luchas del padre de los dioses contra las fuerzas del mal y que no fuesen acontecimientos importantes para su identidad colectiva, ya que se recuerda lo que es importante, y lo que es importante requiere de una conmemoración.

\section{La Xandica y el festival de la Basileia}

Ahora bien, una cosa es comprobar que tanto en Macedonia como en Seleucia la derrota de los gigantes y Tifón tuvo una especial relevancia y otra muy distinta es demostrar que existiese algún acto, rito o festival para celebrarlo. Dada la cantidad de indicios que tenemos podemos sospecharlo, pero no dejará de ser una suposición hasta que aumente nuestro exiguo conocimiento sobre los festivales macedonios.

No obstante, sabemos que pudo existir una fiesta semejante en la frontera entre Macedonia y Tesalia, en Perrebeia. Allí se encontraba la ciudad de Pition ${ }^{87}$ que cada nueve años enviaba a Delfos una procesión para festejar la muerte de Pitón a manos de Apolo. ${ }^{88}$ No se trata ni de un gigante ni de Tifón, pero como ellos fue un ser anguiforme y una encarnación del terror. Esta procesión se aproximaría bastante a lo que estamos buscando, pero se trata de un acto ligado a Delfos, y no a la casa real de Macedonia. Aunque dadas las semejanzas entre el culto tesalio y el macedonio sería sorprendente que no existiese algo similar en Macedonia o no que fuese asimilado tras la conquista de Perrebia por Filipo II.

En primer lugar deberíamos concretar el lugar en el que acontecían los posibles actos de celebración. Díon parece sin duda el candidato más propicio por estar consagrado al dios y a sus hijas las musas, y por su proximidad al monte Olimpo. Sin embargo, no hay que olvidar otros emplazamientos de gran importancia para los macedonios como Egas, la primera capital del reino y desde la que también puede divisarse el Olimpo. ${ }^{89}$ No obstante, el emplazamiento no pudo haber sido una conditio sine qua non, ya que durante los once años que estuvo fuera de Macedonia, Alejandro también tuvo que haber honrado a su divino progenitor.

Existen motivos para pensar que la celebración que estamos buscando podría haber sido la Xandica. Cuando Seleuco ascendió al monte Casio no lo hizo durante un día normal para los macedonios, sino durante el 23 del mes de Xandicos ${ }^{90}$ (abril). Aparentemente en el calendario macedonio los nombres de los meses derivan de festividades religiosas..$^{91}$ En ese mes se celebraba la Xandica. Se trataba de una ceremonia de purificación dirigida por el rey, en la cual el ejército pasaba sobre los restos ensangrentados de una perra descuartizada: ${ }^{92}$

87 Liv. XLII 53. 6-7; Plut. Aemi. 15; Steph. Byz. s.v. ПúӨıov.

88 Fonterose 1959, 88; Mari 2002, 30.

89 LANE Fox 2011, 388.

90 KALLÉRIS 1976, 565-566.

91 KALlÉRIS 1976, 554; MARI 2011a, 460.

92 Sobre otros ejemplos de sacrificios de perros veáse: Plut. Mor. 290d; Paus. III 14.9-10. Cf. LE BoHECBouhet 2011, 503 y ss., ofrece un detallado análisis de la importancia del perro en la Xandica. 
Decidieron hacer una «purificación» del ejército siguiendo la costumbre nacional y la pasada discordia les parecía una razón muy apropiada. Los reyes macedonios solían purificar el ejército de acuerdo con el siguiente ceremonial: mataban una perra y sus vísceras las arrojaban en las dos extremidades de una llanura; en medio era introducido todo el ejército armado, a un lado la caballería, al otro la falange. ${ }^{93}$

\section{T. Livio describe la misma ceremonia en tiempos de Filipo V:}

Precisamente entonces llegó la fecha de la purificación del ejército, cuyo ceremonial consiste en lo siguiente: se corta una perra por la mitad y se coloca la parte de la cabeza en el lado derecho del lugar de paso, y la parte trasera, con las entrañas, en el lado izquierdo. Entre estas dos mitades de la víctima se hace desfilar a las tropas con sus armas. A la cabeza del desfile son portadas las armas y los emblemas de todos los reyes de Macedonia desde sus más remotos orígenes; sigue luego el rey con sus hijos, a continuación desfila la cohorte real y su guardia personal, y cierra la marcha el resto de las tropas de macedonios. ${ }^{94}$

La Xandica se celebraba en primavera durante el equinoccio, y tras la misma los nuevos reclutas pasaban a formar parte del ejército. ${ }^{95}$ Llama la atención que se escogiera a una perra para hacer el sacrificio y que el ejército tuviera que pasar entre sus restos para purificarse. La mitología ofrece un caso muy similar por medio de Peleo, el padre de Aquiles. Cuando éste mató a Astidamía, la despedazó e hizo que su ejército pasase entre sus restos. ${ }^{96} \mathrm{El} \pi \varepsilon \rho ı \sigma \chi v \lambda \alpha \kappa 1 \sigma \mu o ́ \varsigma$ (el descuartizamiento de un perro) pudo haber sido un rito común de tesalios y macedonios.

Pero ante todo era una ceremonia que servía para ensalzar a la casa real de los Argéadas, como refleja que las armas y los emblemas de todos los reyes estuvieran presentes. Más significativo es el papel jugado por los príncipes en el ritual:

Los dos jóvenes hijos del rey lo escoltaban a ambos lados: Perseo, que tenía entonces treinta años, y Demetrio, cinco años más joven; en pleno vigor de la juventud el primero y en la flor de la juventud el segundo, retoños adultos de un padre afortunado si su cabeza hubiese regido bien. Finalizado el rito de la purificación era costumbre que el ejército realizara ejercicios repartiéndose en dos líneas de batalla que se enfrentaban en un simulacro de combate. Los jóvenes príncipes fueron designados como jefes para esta batalla simulada; pero no fue un combate fingido sino que cargaron como si el reino estuviera en juego; se ocasionaron muchas heridas con los palos, y exceptuando el hierro no faltó nada para dar la sensación de una verdadera guerra. La división mandada por Demetrio resultó netamente superior. ${ }^{97}$

93 Curt. X 9.11-12 (traducción de F. Pejenaute Rubio, Biblioteca Clásica Gredos, 1986); Cf. Arr. Succ. 1.4.

94 Liv. XL 6.1-3 (traducción de J. A. Villar Vidal, Biblioteca Clásica Gredos, 1993).

95 Hatzopoulos 1994, 89; Walbank 2002, 80.

96 Apollod. III 13.7. Cf. Hdt. VII 39, Jerjes hizo pasar a su ejército entre el cuerpo descuartizado de una persona.

97 Liv. XL 6.4-7 (traducción de J. A. Villar Vidal, Biblioteca Clásica Gredos, 1993). 
Desconocemos el significado de ese combate simulado y por lo tanto cualquier interpretación es hipotética. ${ }^{98}$ Para Hatzopoulos es un rito de paso semejante a otros que se hacían en Esparta como la sphairomachia, sin embargo, la procesión con los emblemas de la casa real y la destacada presencia de los reyes sugieren que no nos encontramos únicamente ante un simple rito de iniciación. ${ }^{99}$

La Xandica derivaría del héroe Janto/Xanthos, quien luchó contra Melanthos en las fronteras entre Beocia y Ática. ${ }^{100}$ Los nombres Xanthos y Melanthos se relacionan con rubio y negro, luz y oscuridad. Siguiendo esta historia Usener ha defendido que la Xandica representaba un duelo entre el dios de la luz y el verano, y el dios del invierno y la oscuridad. ${ }^{101}$ La teoría es interesante, pero no está claro que el Xanthos ático y el macedonio sean el mismo héroe. Si la Xandica ocurría en primavera es posible que se relacionase con el cambio estacional, con la sucesión entre luz y oscuridad. Si se recuerda la anteriormente citada Musomachia, en la versión de Antonino Liberal (IX), las Piérides representan las tinieblas mientras que las musas combaten bajo el respaldo de la luz:

Entonces, cada vez que se ponían a cantar las hijas de Píero, todo se oscurecía y no se prestaba ninguna atención al coro; sin embargo, al son de las Musas permanecían quietos cielos y astros, mar y ríos, y el Helicón, embelesado, creció lleno de placer hacia el cielo, hasta que por orden de Posidón, Pegaso lo paró golpeando la cima con su pezuña. ${ }^{102}$

Antonino Liberal no menciona ni a los gigantes ni a Tifón, pero sí lo hace Ovidio. Si como pensamos ambos autores resumieron la obra de Nicandro, es posible que en el texto original Tifón y los gigantes fuesen asociados a la oscuridad, mientras que las musas y las deidades olímpicas permanecían del lado de la luz. Luego la Xandica podría estar relacionada con la Musomachia y por extensión con la gigantomaquia y la tifonomaquia.

Todas estas conjeturas no son suficientes para establecer que la Xandica conmemoraba la victoria de Zeus sobre los hijos de Gea. Recapitulemos: 1) El momento de la celebración, el equinoccio, puede ser visto como una celebración de la victoria de la luz frente a la oscuridad 2) Hay una procesión de la casa real en la que las armas y los emblemas de los anteriores monarcas son expuestos, luego la ceremonia es también una exaltación de la familia que detenta el poder 3) Son los miembros de la casa real quienes lideran los bandos enfrentados durante el combate simulado, de tal modo la victoria siempre iría a parar a un miembro de los Argéadas 4) Dada la fiereza con la que ambas facciones peleaban entre sí, podemos inferir que el resultado no estaba pactado, y dado el carácter maniqueo de esta celebración, implicaría que el vencedor

98 Le Bohec-Bounet 2011, 505, señala que el combate debía ser simulado, ya que las tropas purificadas no podían verter la sangre hasta la próxima campaña.

99 Walbank 2002, 80, estudia la Xandica en el contexto de otras procesiones helenísticas.

100 Plb. XXIII 10.17; Helánico FGrH F 125.

101 Usener 1904, 304; Cornford 1934, 67; Kirby 2002, 182-183. Cf. TARn 1928, 197-198, quien lo llama dios de la luz.

102 Traducción de J. R. del Canto Nieto, Akal, Colección Clásicos griegos, 2003. 
era el verdadero representante de la legitimidad y su rival el pretendiente ilegítimo. En suma, la Xandica podría haber tenido una función similar al Akîtu, al fortalecer la posición del monarca, ya que éste quedaba convertido en un campeón del orden cósmico.

Ahora bien, los monstruos que encarnan la maldad no están presentes explícitamente en la Xandica, sólo pueden ser indirectamente relacionados a través de la Musomachia. Tampoco está presente Zeus, cuya persona debería confundirse con la del monarca. El descuartizamiento del perro y el ritual de la Xandica posiblemente también se celebraron en Tesalia ${ }^{103}$ (Perrebeia). El calendario de la Xandica, tampoco da fuerza a nuestra hipótesis, pues el mes en el que los macedonios rendían homenaje al padre de los dioses, era Dios (octubre) y no el mes de Xandos (abril). El calendario macedonio comenzaba precisamente en el mes de Dios, mientras que el babilonio lo hacía en el mes de Nisán (marzo-abril).

Se cree que los persas también habrían celebrado su año nuevo en fechas similares a las de los macedonios (octubre), pero que su gran festividad el Now Ruz habría sido cambiada por la dinastía Sasánida. ${ }^{104}$ Debemos recordar que durante casi 3 décadas Macedonia fue un reino sometido a la voluntad del Gran Rey. Incluso Jerjes estuvo un tiempo en Macedonia durante la invasión persa de Grecia. Si como se sospecha el Now Ruz emulaba al Akîtu, los macedonios podrían haber imitado el ritual de los persas, como otros aspectos relacionados con su monarquía. ${ }^{105}$ Sin embargo, ni estamos seguros de que el Now Ruz fuese un equivalente exacto del Akîtu, ${ }^{106}$ ni sabemos a ciencia cierta que el cambio de fecha tuviera lugar. Por otro lado, la impronta de los hijos de Gea en Macedonia era tan grande que no parece necesario recurrir a influencias extranjeras para ser conscientes de su potencial.

Existe una alternativa a la Xandica y es el festival de la Basileia. Estaba dedicado a Zeus Basileus. Se cree que es la deidad a la que Alejandro sacrificó en Díon ${ }^{107}$ o bien en Egas antes de partir a Asia. ${ }^{108}$ Los macedonios identificaron a esta manifestación de Zeus en Gordión y confirmaron sus sospechas al ver rayos poco después. Alejandro volvió a sacrificar a este dios en Menfis en el 331 a.C. ${ }^{109}$ Al parecer las celebraciones de este festival estaban acompañadas por juegos atléticos, muy comunes en el pueblo macedonio. ${ }^{110}$ Más tarde en el Egipto de los Ptolomeos se combinó esta fiesta con las celebraciones por el aniversario del monarca, siendo el caso más célebre el de Ptolomeo II Filadelfo. Conocemos por una inscripción (IG II $\left.{ }^{2} 3779\right)$ que durante los festejos se leían himnos especialmente compuestos para honrar a la deidad y al soberano. Se cree que el himno a Zeus de Calímaco y el Heracliscus de Teócrito pudieron haber sido escritos con ocasión del festival de la Basileia. ${ }^{111}$ Un himno compuesto en

103 Grainger 2011, 113.

104 Boyce 1982 II 109; ID. 1983, 801-802; SAnCISI-WeErdendurg 1991.

105 Goukowsky I 1978, 11-12; Olbrycht 2010, 345.

106 García SÁnchez 2008, 21.

107 Plut. Alex. 14.8-9.

108 Arr. An. I 11.1.

109 Gordión (Arr. An. I 17.6); Menfis (Arr. An. III 1.4; 5.2).

110 Adams 2003; ID. 2007.

111 Acosta-Hughes - Stephens 2012. 
honor a Zeus habría sido una forma sencilla de presentar al monarca como un mediador entre los dioses y la comunidad, y de convertir a sus oponentes en enemigos del propio dios. ${ }^{112}$ Sin embargo, las posibilidades de encontrarnos con una versión canónica de un himno macedonio a Zeus serían muy altas, y no tenemos algo semejante. Seguimos en definitiva sin evidencias que demuestren la existencia de una fiesta en Macedonia semejante al Akîtu.

También sigue siendo un problema la falta de fuentes que certifiquen que los reyes macedonios asociaban a sus enemigos con los de Zeus antes de época helenística. El debate que planteamos no puede cerrarse sin más datos. Sin embargo, queremos presentar una posible evidencia, se trata de una moneda de Amintas III, el padre de Filipo II. En ella se aprecia la imagen de Heracles en el anverso y en el reverso un águila devorando a una serpiente. No hay una interpretación clara sobre la misma, ${ }^{113}$ pero parece haberse acuñado para conmemorar la victoria de Amintas sobre los enemigos que le habían destronado (Diod. XIV 92.3). Lo que implicaría que el águila, símbolo por excelencia de Zeus, y Amintas debían ser el mismo personaje representado en la moneda. Por lo tanto, es justo preguntarse si el enemigo de Zeus-Amintas es simplemente una serpiente, o son los seres que quisieron destronar a ambos reyes, ya que tanto Tifón como los gigantes eran seres anguiformes. Amintas podría haber iniciado el camino para separar la figura del rey de la del resto de sus súbditos, un proceso que finalizaron su hijo y nieto. Sabemos que su reinado no fue fácil y que fue destronado al menos en una ocasión, por lo que estos actos propagandísticos habrían sido muy útiles para fortalecer su posición.

\section{Conclusión}

Resulta llamativa la facilidad que algunos investigadores atribuyen a los macedonios, y a Alejandro en particular, para adoptar nuevas costumbres funerarias, vestimentas o creencias religiosas tras la conquista de Asia. En parte, esta idea viene de la creencia asumida de que Alejandro cambió el mundo, ${ }^{114}$ pero también se basa en la salvedad que la sociedad macedonia fue sumamente primitiva y descentralizada hasta los tiempos de Filipo II. Es innegable que se trató de una comunidad menos civilizada que la ateniense y carente de formas institucionales tan desarrolladas y complejas como la democracia, pero su monarquía estuvo lejos de ser "simple". Los Argéadas desarrollaron estructuras de legitimación de una elaboración sumamente destacable. Dada su ascendencia heráclida, su progresiva identificación con Zeus, la importancia

112 Sirva de ejemplo este pasaje del mencionado Himno a Zeus de Calímaco (79-84): "pero los reyes vienen de Zeus, y nada hay más divino que los reyes de Zeus: por eso hiciste de ellos tu parte. Les confiaste la guarda de las ciudades, y tú, en lo alto de la ciudadela, estás sentado, atento a quién gobierna al pueblo con medios tortuosos y a quién lo hace con justicia” (traducción de L. A. de Cuenca y Prado y M. Brioso Sánchez, Biblioteca Clásica Gredos, 1980).

113 HAMmOND - GRIFFITH 1979, 180: "The emblems on the coins of Amyntas III stress the claim of the new line of the royal family to be descended from Heracles: his head, his club, and the wild boar he slew figure most prominently. An eagle standing on and striking a snake is a new emblem".

114 Droysen 1833, 3: "Der Name Alexander bezeichnet das Ende einer Weltepoche, den Anfang einer neuen". 
del Olimpo para los macedonios y la huella de los gigantes y de Tifón en Macedonia ¿resulta inconcebible que conmemorasen la victoria de Zeus sobre los hijos de Gea? Lo es desde algunas concepciones que entienden al monarca como un mero conductor de hombres, limitado por la asamblea y el ejército. ${ }^{115}$ Ahora bien, una dinastía que ha conseguido perpetuarse en el poder más de dos siglos, pese al elevado número de regicidios, ${ }^{116}$ no lo consigue sin antes haber desarrollado un carisma regio que distinga y diferencie a sus miembros del resto de sus súbditos. ${ }^{117}$ Este carisma se cimienta no sólo en el derecho de conquista, sino ante todo en el linaje divino de los Argéadas. ¿Podía el clan que gobernaba el reino pasar por alto dos de las mayores gestas que sus antepasados Heracles y Zeus habían realizado en su propia tierra? Carecemos de las evidencias necesarias para constatar nuestra teoría, pero a veces es más importante plantear una pregunta que dar una respuesta.

\section{Bibliografía}

Acosta-Hughes, B. - Stephens, S. A. (2012): Callimachus in Context: From Plato to the Augustan Poets, Cambridge.

AdAms, L. W.

(2003): "Other Peoples Games: The Olympics, Macedonia and Greek Athletics”, JSH 30/2, 205-217.

(2007): "The Games of Alexander the Great", [en] W. Heckel - L. Tritle - P. Wheatley (eds.), Alexander's Empire: Formulation to Decay, Claremont, 25-38.

ANTEla BERnÁRDEZ, B.

(2007): “Alejandro Magno o la demostración de la divinidad", Faventia 29/1, 89-103.

(2009): "Ciudad y territorio en la Siria helenística. Conquista, control y redifinición desde Seleuco Nicator", [en] B. Antela Bernárdez - T. Naco del Hoyo (eds.), Transforming Historical Landscapes in the Ancient Empires (=BAR International Series 1986), Oxford, 119-130.

ArenA, E. (2013): “Alessandro basileus nella documentazione epigrafica: la dedica del tempio di Atena a Priene (I.Priene 156)", Historia 62, 48-80.

Aymard, A. (1950): "Sur l'assemblée macédonienne”, REA 52, 115-137.

BernARD, P. (1990): "Nouvelle contribution de l'épigraphie cunéiforme a l'histoire hellenistique", $B C H 114,513-541$.

Billows, R. (1995): Kings and Colonists: Aspects of Macedonian Imperialism, Leiden.

BorzA, E. N. (1990): In the shadow of Olympus: The Emergence of Macedon, Princeton.

Bosworth, A. B. (1996): Alexander and the East. The Tragedy of Triumph, Oxford.

115 Granier 1931; Aymard 1950.

116 La lista de los reyes macedonios asesinados es ingente. Alcetas II, Arquelao, Orestes, Amintas II, Pausanias I, Alejandro II y Filipo II son algunos ejemplos, pero pese a la extensión del regicidio, el poder nunca abandonó a los Argéadas. Cf. CARNEY 1983, 260-272.

117 Cf. Molina Marín 2014a. 
Boyce, M.

(1982): A History of Zoroastrianism: Volume II: Under the Achaemenians, Leiden.

(1983): "Iranian Festivals", [en] E. Yarshater (ed.), The Cambridge History of Iran III/2, Cambridge, 792-819.

Burstein, S. M. (1985): The Hellenistic Age from the Battle of Ipsos to the Death of Kleopatra VII (=Translated Documents of Greece and Rome 3), Cambridge.

Callaghan, P. J. (1981): "On the date of the great altar of Zeus at Pergamon", BICS 28/1, 115-121 (http://dx.doi.org/10.1111/j.2041-5370.1981.tb00422.x).

CARney, E. (1983): “Regicide in Macedonia”, PP 38, 260-272.

Clauss, J. J. (1986): "Lies and Allusions: The Addressee and Date of Callimachus' "Hymn to Zeus"”, CA 5/2, 155-170 (http://dx.doi.org/10.2307/25010845).

Cohen, G. M. (2006): The Hellenistic Settlements in Syria, the Red Sea Basin and North Africa, Berkeley.

Cornford, F. M. (1934): The Origin of Attic Comedy, Cambridge.

DAHMEN, K. (2007): The legend of Alexander the Great on Greek and Roman coins, New York.

Dowden, K. (2006): Zeus (Gods and Heroes of the Ancient World), London-New York.

Downey, G. (1961): A History of Antioch in Syria: from Seleucus to the Arab conquest, Princeton.

Droysen, J. G. (1833): Alexander der Grosse, Berlin.

EdDy, S. K. (1961): The king is dead: studies in the Near Eastern resistance to Hellenism, 334-31 B.C., Lincoln.

EDMunds, L. (1971): “The Religiosity of Alexander”, GRBS 12, 363-391.

Edson, CH.

(1934): "The Antigonids, Heracles, and Beroea", HSCP 45, 213-246 (http://dx.doi. org/10.2307/310636).

(1948): "Philip V and Alcaeus of Messene", CPh 43/2, 116-121 (http://dx.doi. org/10.1086/363091).

ENGELS, J. (2010): “Macedonians and Greeks", [en] J. Roisman - I. Worthington (eds.), A Companion to Ancient Macedonia, Oxford, 81-98.

ERICKSON, K.

(2009): The early Seleucids, their gods and their coins, Ph.D. diss., University of Exeter. (2011): "Apollo-Nabû: The Babylonian policy of Antiochus I", [en] K. Erickson - G. Ramsey (eds.), Seleucid Dissolution: The Sinking of the Anchor (=Philippika 50), Wiesbaden, 51-66.

Errington, M. (1990): A History of Macedonia, Berkeley-Los Angeles.

Fennelly, J. M. (1980): "The Persepolis ritual”, BiA 43, 135-162 (http://dx.doi. org/10.2307/3209721).

Fonterose, J. (1959): Python. A study of Delphic myth and its origins, Berkeley-Los Angeles.

Fredricksmeyer, E. A. (2000): "Alexander and the Kingship of Asia”, [en] A. B. Bosworth E. J. Baynham (eds.), Alexander the Great in Fact and Fiction, Oxford, 136-166.

Gaebler, H. (1935): Die antiken Münzen Nord-Griechenlands, III. Makedonia und Paionia, Berlin.

García SÁnchez, M. (2008): “Persépolis, ¿arquitectura celestial o terrenal?”, Historiae 5, $11-25$. 
GirTzy, M. (2001): Historical topography of Ancient Macedonia, Thessaloniki.

Goukowsky, P. (1978-1981): Essai sur les origines du mythe d'Alexandre, I-II, Nancy.

GRAINGER, J. D.

(1990): The cities of Hellenistic Syria, Oxford.

(2011): Cult and Koinon in Hellenistic Thessaly, Leiden-Boston.

Granier, F. (1931): Die makedonische Heeresversammlung. Ein beitrag zum antiken Staatsrëcht (=Münchener Beiträge zur Papyrusforschung und antiken Rechtsgeschichte 13), München.

Hammond, N. G. L. - Griffith, G. T. (1979): A History of Macedonia. Volume II 550-336 B.C., Oxford.

Hardie, Ph. R. (1986): Virgil's Aeneid: cosmos and imperium, Oxford.

Harrington, S. P. M. (1996): "Sanctuary of the Gods", Archaeology 49/2, 28-35.

Hatzopoulos, M. I. (1994): Cultes et rites de passage en Macedoine, Athens.

Heisserer, A. J. (1980): Alexander the Great and the Greeks. The Epigraphic Evidence, Norman, Oklahoma.

Holt, Fr. L. (2003): Alexander the Great and the mystery of Elephant Medallion, BerkeleyLos Angeles.

Johnson, P. - Malamud, M. (1988): “Ovid's “Musomachia””, PCP 23/1-2, 30-38 (http:// dx.doi.org/10.2307/1316682).

Kalléris, J. N. (1976): Les Anciens Macédoniens. Étude linguistique et historique, II, Athens. Kertész, I. (1993): “The Attalids of Pergamon and Macedonia”, Archaia Makedonia V, 669-677.

Kirby, E. T. (2002): “Greece: The forms of Dionysus”, [en] H. Bloom (ed.), Greek drama, Broomall, 169-210.

Koepr, F. (1888): De Gigantomachie in poeseos artisque monumentis usu, Bonn.

LANE FOX, R.

(2008): Travelling Heroes: Greeks and Their Myths in the Epic Age of Homer, London.

(2011): "Philip's and Alexander's Macedon", [en] Lane Fox (ed.), 2011, 367-391.

Lane Fox, R. (ED.), (2011): Brill's Companion to Ancient Macedon. Studies in the Archaeology and History of Macedon, $650 \mathrm{BC}-300 \mathrm{AD}$, Leiden-Boston.

Le BoHec-Bounet, S. (2002): "The kings of Macedon and the cult of Zeus in the Hellenistic period", [en] D. Ogden (ed.), The Hellenistic world: new perspectives, Swansea, 41-57.

(2011): "Les chiens en Macédoine dans l'Antiquité", [en] N. Badoud (éd.), Philologos Dionysios: mélanges offerts au professeur Denis Knoepfler, Genève, 491-515.

Le Rider, G. (2007): Alexander the Great. Coinage, Finances and Policy, Philadelphia.

LiAMPI, K. (1998): Der Makedonische Schild, Bonn.


ral, Aristotle University of Thessaloniki.

Manunta, E. (2008): “Giganti sull'acropoli. Atene, Pergamo e la Gigantomachia”, Lanx 1, 75-109.

MARI, M.

(2002): Al di là dell'Olimpo. Macedoni e grandi santuari della Grecia dall'età arcaica al primo ellenismo, Athens. 
(2011): “Archaic and Early Classical Macedonia”, [en] Lane Fox (ed.), 2011, 79-92. (2011a): "Traditional Cults and Beliefs", [en] Lane Fox (ed.), 2011, 453-476.

Mayor, A. (2000): The First Fossil Hunters: Paleontology in Greek and Roman Times, Princeton.

Molina Marín, A. I.

(2012): Geographica: ciencia del espacio y tradición narrativa de Homero a Cosmas Indicopleustes (=Antigüedad y Cristianismo 27), Murcia.

(2012a): Alejandro Magno. Apotheosis y Paideia. La figura del gobernante entre el homenaje y la teoría política, Madrid.

(2014): "La fascinación por el gigantismo en mundo helenístico. El elefante de guerra", [en] O. Olesti - J. Vidal - B. Antela (eds.) Animales de guerra en el mundo antiguo, Zaragoza, 73-91.

(2014a): "El miedo como arma de dominación: Admiración, pavor y victoria", Gladius 35, 95-110 (http//dx.doi.org/10.3989/gladius.2014.0004).

Momigliano, A. (1942): “Terra marique”, JRS 32, 53-64 (http://dx.doi.org/10.2307/296460).

Mørкholm, O. (1991): Early Hellenistic Coinage from the Accession of Alexander to the Peace of Apamea (336-186 B.C.), Cambridge.

Nock, A. D. (1930): “Synnaos Theos”, HSPh 41, 1-62.

OGDEN, D.

(2011): Alexander the Great: Myth, Genesis and Sexuality, Exeter.

(2013): Drakon: Dragon Myth and Serpent Cult in the Greek and Roman Worlds, Oxford. (2013a): Dragons, Serpents, and Slayers in the Classical and Early Christian Worlds: A Sourcebook, Oxford.

Palagia, O. (2011): “Hellenistic Art”, [en] Lane Fox (ed.), 2011, 477-493.

Pandermalis, D. (1997): Dion, the archaeological site and the museum, Athens.

Pearson, L. I. C. (1960): The Lost Histories of Alexander the Great, New York.

Pingiatoglou, S. (2010): "Cults of Female Deities at Dion”, Kernos 23, 179-192 (http:// dx.doi.org/10.4000/kernos.1578).

Pollitt, J. J. (1989): El arte helenístico, Madrid.

Ridgway, B. S. (2001): Hellenistic sculpture I. The styles of ca. 331-200 B.C., Madison, Wis. SAncisi-Weerdenburg, H. (1991): "Nowruz in Persepolis", AchHist VII, 173-201.

STEWART, A.

(1993): Faces of Power: Alexander's Image and Hellenistic Politics, Berkeley-Los Angeles. (2000): "Pergamo Ara Marmorea Magna. On the date, reconstruction, and functions of the Great Altar of Pergamon", [en] N. T. de Grummond - B. S. Ridgway (eds.), From Pergamon to Sperlonga: Sculpture and Context, Berkeley-Los Angeles, 32-57.

TARN, W. W.

(1928): "Macedonia and Greece", [en] CAH VII, 197-223.

(1938): The Greeks in Bactria and India, Cambridge.

Tripodi, BR. (1986): “L’emblema della casa reale macedone”, Archaia Makedonia IV, 653660.

Trofimova, A. (2012): "Alexander and the Giants of the Pergamon Frieze", [en] Imitatio Alexandri in Hellenistic Art: Portraits of Alexander the Great and Mythological Images (=L’Erma di Bretschneider, Studia Archaeologica 187), Roma, 125-132. 
Usener, H. (1904): “Heilige Handlung”, Archiv für Religionswissenschaft VII, 281-339.

WALBANK, F. W.

(1940): Philip V of Macedon, Cambridge.

(1944): "Alcaeus of Messene, Philip V, and Rome: A Footnote", CQ 38/3-4, 87-88 (http:// dx.doi.org/10.1017/S0009838800030391).

(2002): Polybius, Rome and the Hellenistic World: Essays and Reflections, Cambridge.

Whitaker, R. (2005): “Art and ideology: The case of the Pergamon gigantomachy", $A C 48$, 163-174. 